\title{
Reducing Perioperative Neurocognitive Disorders (PND) Through Depth of Anesthesia Monitoring: A Critical Review
}

This article was published in the following Dove Press journal: International Journal of General Medicine

\author{
Lisbeth A Evered ${ }^{1-4}$ \\ Peter A Goldstein ${ }^{1,4,5}$ \\ 'Department of Anesthesiology, Weill \\ Cornell Medicine, New York, NY, USA; \\ ${ }^{2}$ Department of Anaesthesia and Acute \\ Pain Medicine, St. Vincent's Hospital, \\ Melbourne, VIC, Australia; ${ }^{3}$ Department \\ of Medicine, University of Melbourne, \\ Melbourne, VIC, Australia; ${ }^{4}$ Feil Family \\ Brain and Mind Research Institute, Weill \\ Cornell Medicine, New York, NY, USA; \\ ${ }^{5}$ Department of Medicine, Weill Cornell \\ Medicine, New York, NY, USA
}

\begin{abstract}
General anesthesia has been administered for over 150 years, and in that time, has become progressively safer. Improvements in outcomes have been driven by multiple advances, including the use of non-invasive monitors to assess cardiovascular and respiratory status. More recent advances have included the development and use of monitors to measure neurologic status by means of "processed" electroencephalography (pEEG), wherein the frontal EEG signal is analyzed by proprietary algorithms to produce a dimensionless number (scaled from 0 to 100), wherein low values are associated with deepening levels of sedation that progresses to loss of consciousness. Such monitors have been shown to enable anesthetic titration so as to expedite emergence and early recovery, and their use is advocated for the prevention of intraoperative awareness in the setting of administration of total intravenous anesthesia and neuromuscular blockade. Whether their use can minimize, or prevent, longer term adverse events is a matter of debate. In this narrative review of the most recent literature, we provide an assessment on the use of pEEG monitors in the prevention of a notable, and important, postoperative adverse outcome - delirium - in elderly patients. As we will discuss, the existing data do not support its routine use for the prevention of postoperative delirium in this, or any other, patient population.
\end{abstract}

Keywords: delirium, outcome, postoperative, EEG, electroencephalography

\section{Introduction}

Moller et al published their landmark study on long-term postoperative cognitive dysfunction (POCD) in the elderly in 1998. ${ }^{1}$ A Boolean search in Pubmed (terms: postoperative, cognitive, dysfunction, elderly) returns 2252 articles published from 1998 until now covering preclinical and clinical investigations, review articles, meta-analyses, and guidelines, indicating significant, ongoing interest in the topic. POCD, though, is a term that is imprecise, and within the field, there is recognition that a more rigorous nomenclature is required so as to clarify the problem under investigation, thereby improving our ability to study the phenomenon in a more rational manner and diagnose clinically. ${ }^{2}$ The Nomenclature Consensus Working Group authors highlighted a number of important issues, not the least of which is the need to distinguish between delirium (an acute, self-limiting pattern of disorganized thinking with fluctuations in attention, awareness, and consciousness, which may be associated with either hyper- or hypo-activity) and neurocognitive disorders (which can be short term, occurring up to 30 days after the procedure and should be classified as delayed neurocognitive recovery, or more protracted, occurring up to
Correspondence: Peter A Goldstein Department of Anesthesiology, Weill Cornell Medicine, New York, NY 10065 USA

Tel +I-2I2-746-5325

Email pag20I4@med.cornell.edu
International Journal of General Medicine 2021:|4 I53-162 
12 months post-procedure, which should be classified as postoperative neurocognitive disorder). ${ }^{2}$ It is routinely assumed that if a patient experiences any decline in cognitive function in the postoperative period that it must, on its face, be the result of anesthetic exposure since general anesthetics, whether intravenous or volatile, so clearly perturb cognitive function. If this is true, then successfully monitoring anesthetic depth should facilitate the accurate titration of anesthetic administration, thereby preventing unintentional over-exposure to potentially toxic drugs. But when considering the question of depth of anesthesia monitoring in elderly patients, what is it that we are hoping to achieve? Is it prevention of postoperative delirium, or are we looking to prevent, or attenuate, a more pernicious decline in cognitive function? Are such goals, while clearly laudable, achievable? Here we hope to address these questions.

\section{Perioperative Neurocognitive Disorders}

It has long been acknowledged that many older individuals undergoing anesthesia and surgery are "not the same" postoperatively. Perioperative Neurocognitive Disorders (PND) encompass cognitive impairment existing preoperatively, postoperative delirium (POD), delayed neurocognitive disorder (dNCR), and postoperative neurocognitive disorder (NCD). $\mathrm{dNCR}$ and postoperative NCD align with mild cognitive impairment ((MCI) - mild NCD)) and dementia (major NCD). ${ }^{2}$ More than $20 \%$ of older individuals undergoing anesthesia and surgery will experience new $\mathrm{MCI}^{3}$ and up to $30 \%$ of patients will experience worsening dementia. ${ }^{4}$

A major issue when considering meta-analyses of studies investigating $\mathrm{pEEG}$ and POD is the heterogeneity of the tests used to assess for postoperative delirium. Many, such as the $\mathrm{CAM},{ }^{5} \mathrm{CAM}-\mathrm{ICU},{ }^{6}$ and 3-minute diagnostic CAM (3D-CAM), ${ }^{7}$ are based on the Diagnostic and Statistical Manual of Mental Disorders (DSM-IV or DSM-5) and have similar sensitivity and specificity when used on the appropriate population of people and with appropriate training. Other tools include the Delirium Rating Scale - Revised-98 (DRS-R98), the Memorial Delirium Assessment Scale (MDAS), and the Nursing Delirium Screening Scale (nu-DESC). Evidence from an assessment of various delirium tools concluded the best evidence supported the use of the CAM. ${ }^{8}$ Subsequent to this publication the 3D-CAM has been developed with similar sensitivity and specificity, it is a more structured tool with excellent online training and takes only 3 minutes to administer.

\section{Postoperative Delirium}

The incidence of postoperative delirium (POD) varies with the type of surgery performed; it can be as low as $4 \%$ in older individuals undergoing cataract surgery and as high as $65 \%$ in individuals undergoing hip arthroplasty following fracture. ${ }^{9}$ Early PND, particularly delirium, are associated with a significant risk of short and longer-term complications. It is estimated that at least $10.6 \%$ of community dementia is the direct result of delirium, indicating prevention of delirium will likely reduce the prevalence of dementia. $^{10}$

Delirium is a neurocognitive disorder associated with an acute and fluctuating change in attention, awareness, and consciousness. ${ }^{11}$ It occurs in three types, hypoactive, hyperactive, and mixed. ${ }^{11}$ It is believed hypoactive delirium is more common than any other type and is estimated to go undetected in up to $60 \%$ of hospitalized patients ${ }^{11}$ due to its presentation, which may include fatigue, apathy, and sleepiness.

Delirium is associated with an increase in length of hospital stay, unexpected admission to the intensive care unit (ICU), institutionalization, postoperative complications, progression to dementia, mortality, and morbidity. ${ }^{8}$ POD is also associated with long-term psychosocial impairment, including distress, isolation, and psychological trauma. ${ }^{12}$ Reducing the incidence of POD has the potential to improve postoperative outcomes for older individuals, improve recovery, improve independence, and reduce the community burden of dementia.

\section{New Cognitive Impairment}

As mentioned above, "new" cognitive impairment, as distinct from POD, occurs in up to $20 \%$ of individuals aged 65 years or older up to 12 months or more following anesthesia and surgery. ${ }^{3}$ The definition of "new" is used because patients present to hospital preoperatively as independent individuals, signing their own surgical consent, and having no previous diagnosis of cognitive impairment. However, we know from a number of studies that approximately $20-50 \%$ of these individuals have subtle cognitive impairment preoperatively which is only detected if appropriate neuropsychological tests are undertaken. ${ }^{3,13}$ This poses an important question: Is cognitive decline that occurs in the postoperative period, including POD, 
initiated or exacerbated by the anesthesia and surgery, or is this the cognitive trajectory the individual was already on? This is particularly relevant to POD because preoperative cognitive impairment, even subtle impairment, is associated with a greatly increased risk of POD. ${ }^{14}$

\section{Accelerated Decline in Patients with Underlying Dementia}

Pre-existing dementia is a leading predictor of POD, and an episode of POD is associated with an increased risk of dementia. ${ }^{15}$ This complex association makes it extremely difficult when assessing these individuals during their hospital admission. Delirium superimposed on dementia is difficult to detect because of the overlap in symptoms. The delirium often goes undiagnosed or misinterpreted as an exacerbation of dementia symptoms. ${ }^{15}$ It is difficult to ascertain if any cognitive disruption is the result of POD or dementia, especially given that in general patients are not screened for cognitive function preoperatively.

\section{Death}

Delirium is known to be associated with a significantly increased risk of mortality. ${ }^{14}$ In patients admitted to postacute care their risk of 6-month mortality is 5-fold. ${ }^{11}$ There have been limited studies assessing mortality specifically following POD. A recent study investigating $p E E G$ to reduce POD did find an association between $\mathrm{pEEG}$ guided anesthesia and a reduction in 30-day mortality, but no association was observed between POD and mortality. ${ }^{16}$ Other studies have shown an association with mortality following POD as far as 12 months following surgery. ${ }^{14}$ Large, prospective trials are required to identify if the observed increase in mortality in general medical, ICU, and geriatric patients who experience delirium ${ }^{11}$ is similarly increased following POD.

\section{Depth of Anesthesia Monitoring}

Electroencephalography was first used to describe different planes of anesthetic depth in $1937 .{ }^{17}$ Subsequently, it was demonstrated that in the presence of general anesthetics that the electroencephalographic oscillatory activity was organized as a function of frequency. ${ }^{18,19}$ This organized electrical activity can be displayed in a variety of ways, notably as a compressed spectral array (spectrogram) ${ }^{20}$ which can present the data as a threedimensional plot (power by frequency vs $t^{2} e^{21}$ ) or in two-dimensions as a density-modulated (or density spectral) array. ${ }^{22}$ From these early devices arose the monitors in use today.

Monitored anesthesia utilizing processed electroencephalography (pEEG) has become much more common since the Bispectral Index (BIS) monitor (originally from Aspect Medical Systems, Norwood, MA) was approved by the Federal Drug Administration (FDA) in 1996. Depth of anesthesia monitors, including the BIS (now marketed by Medtronic; Minneapolis, MN) and Sedline (Masimo, Inc.; Irvine, CA), use patented algorithms to "summarize" pEEG to produce a single number between zero and 100 . It is believed that the lower the number, the deeper the anesthesia. Older patients, who are at greatest risk of POD, are also at increased risk of burst suppression within the 40-60 BIS range, generally considered the range for general anesthesia to be maintained. It is thought that burst suppression may be associated with $\mathrm{POD},{ }^{23}$ but whether this is dependent on time of exposure is unclear.

\section{Guidelines}

Although the literature remains controversial, it is interesting to note expert panel publications support the routine use of pEEG monitoring, despite acknowledging it may be of no benefit in reducing the incidence of POD. In 2018 Berger et al published a "best practices for brain health" following the 5th International perioperative neurotoxicity workshop. ${ }^{24}$ The authors state there is insufficient evidence to support pEEG in reducing delirium, and yet go on to conclude there is strong support for pEEG monitoring to reduce POD. In 2019 the American Society of Anesthesiologists Brain Health Initiative published the outcomes of their summit. Their summary of pEEG studies reflected the controversial state of the literature, such that it was not possible to clearly identify a benefit of pEEG monitoring reducing POD. ${ }^{25}$ Despite this, they went on to say "older patients might uniquely benefit .... provided by the EEG spectrogram". This reflects the uncertainty in the anesthetic community regarding the benefit of pEEG to reduce delirium.

\section{Current Evidence from Clinical Trials}

Between 2010 and 2014, a series of randomized control trials (RCTs) examining the relationship between depth of anesthesia (as measured by BIS, or in one instance, auditory evoked potentials - AEPs) and the incidence of postoperative delirium were published. ${ }^{26-29}$ These were 
a diverse group of trials, which included patients undergoing a wide range of surgical procedures (ENT, major noncardiac, and cardiothoracic), variable age range $(>18$, 40-94, >60 years), 2-log order differences in sample size, different measures of postoperative delirium (CAM: Confusion Assessment Method; CAM-ICU: Confusion Assessment Method for the Intensive Care Unit; DSM IV; Diagnostic and Statistical Manual of Mental Disorders IV), and variable follow-up periods (from 1 day only postoperatively to up to day 10 or ICU discharge). Despite their seeming heterogeneity, when analyzed as a group (cumulative sample size $n=1209$ ), the incidence of delirium was lower in monitor-guided subjects than in those who received non-guided anesthetic management (relative risk $0.70,95 \%$ confidence interval (CI), 0.60-0.83; P < 0.0001). ${ }^{30}$ Collectively, the results of these studies suggested that a minimal risk intervention, the use of a simple monitor (BIS or AEP), could reduce the incidence of delirium, an important postoperative complication, one that is associated with significant $\operatorname{costs}^{31}$ and morbidity. ${ }^{32,33}$ But this assessment was soon to be challenged.

In 2018 and 2019, two important prospective clinical trials were published in which the utility of the processed EEG (pEEG) was considered in relationship to the risk of developing postoperative delirium. ${ }^{16,34}$ The Strategy to Reduce the Incidence of Postoperative Delirium in Elderly Patients (STRIDE) study, ${ }^{34}$ published in 2018, was a single-site, double-blind, RCT that enrolled subjects (age $\geq 65 \mathrm{yr}$ ) who were undergoing non-elective hip fracture repair with spinal anesthesia and propofol sedation; subjects were ineligible for participation if delirium, dementia, severe chronic obstructive pulmonary disease, or congestive heart failure were pre-existing conditions. Five hundred and thirty-eight subjects were screened, of which 200 were randomized to receive sedation targeted to a modified observer's assessment of alertness/sedation score (OAA/S) of 0-2 ("heavy sedation"; $n=100$ ) or 3-5 ("light sedation"; $\mathrm{n}=100$ ); a BIS Brain Monitoring System (https://www.medtronic.com/covidien/en-us/pro ducts/brain-monitoring) was used to assess electroencephalographic electrical activity. The diagnosis of delirium was made by a multidisciplinary consensus panel based on DSM-IV criteria using several data sources, including the CAM, the Delirium Rating Scale-Revised-98 (DRS), digit span, a review of medical records, and family/nursing staff interviews.
There was robust separation between the groups (OAA/S: $0.2 \pm 0.4$ vs $4.1 \pm 0.9$, heavy vs light sedation, respectively; BIS: $57.0 \pm 14.8$ vs $82.3 \pm 9.4$, heavy vs light sedation, respectively). The overall incidence of delirium within 1 to 5 days following surgery was $36.5 \%(\mathrm{n}=73)$; in the heavy sedation group, the incidence was 39\% ( $\mathrm{n}=$ 39) while in the light sedation group the incidence was $34 \%(\mathrm{n}=34)$; the difference was not significant $(\mathrm{P}=0.46$, $\chi^{2}$ analysis). Perhaps counter-intuitively, when riskstratified for preexisting comorbidities (as measured by the Charlson Comorbidity Index, CCI), subjects with a $\mathrm{CCI}=0$ (signifying a low level of comorbidity), heavy sedation doubled the risk of developing postoperative delirium (hazard ratio, 2.3; 95\% CI, 1.1.-4.9); in contrast, those subjects with a $\mathrm{CCI}=1-3$ (indicating a higher level of comorbidity), the level of sedation did not alter the risk.

So while it might appear that the use of a pEEG monitor has value, the study did not compare the incidence of delirium as a function of observer rated-sedation to BISmeasured sedation, and given that both assays provided a clear separation between the groups, it is possible that the two approaches are indistinguishable in their ability to predict who is at risk. But the study was not of general anesthesia per se, although the deep level of sedation achieved as indicated by the BIS value would certainly be consistent with a level of unresponsiveness that would equate to that seen during general anesthesia; the issue of general anesthesia was explicitly tested in the next large RCT to be published.

The Electroencephalography Guidance of Anesthesia (ENGAGES) study was published in 2019. ${ }^{16}$ The ENGAGEs study was a randomized trial to assess whether EEG-guided administration of general anesthesia in adults (age $>60 \mathrm{yr}$ ) undergoing major surgery (including cardiac, gastrointestinal, thoracic, gynecologic, hepatobiliarypancreatic, urologic, and vascular procedures) decreased the incidence of postoperative delirium on days 1 to 5 following surgery. Thirty-nine thousand one hundred and forty-four subjects were screened, of which 1400 were enrolled, and 1232 were randomized to receive either EEGguided (Bispectral Index Quatro Bispectral Index Quatro; Medtronic) anesthesia care $(n=614)$ or routine anesthesia care $(n=618)$. The two groups were well-matched across an extensive range of demographic variables, and the overall intraoperative management (with regards to median duration of anesthesia well as doses of midazolam, propofol, opioids, and neuromuscular blocking agents). Delirium was measured using CAM, which is well-suited to detect both 
hypo-and hyper-active delirium. ${ }^{35}$ As might be expected, subjects in the EEG-guided group had lower median endtidal volatile anesthetic concentrations $(0.69$ vs 0.80 minimum alveolar concentration; difference -0.11 [95\% CI, -0.13 to -0.10$])$, less time spent in EEG burst suppression ( 7 vs $13 \mathrm{~min}$; difference -6.0 [95\% CI, -9.9 to -2.11$]$ ), and less time with BIS values $<40$ (32 vs $60 \mathrm{~min}$; difference -28.0 [ $95 \%$ CI, -38.0 to -18.0$]$ ]). The incidence of delirium between the groups was comparable $(n=157(26.0 \%)$ in the EEG group vs $\mathrm{n}=140(23.0 \%)$ in the usual care group (difference $3.0 \%$ [ $95 \% \mathrm{CI},-2.0$ to $8.0 \%, \mathrm{P}=0.22]$ ). Unlike the STRIDE study, the ENGAGES study did not detect evidence that EEG-guided anesthesia care provided any benefit with regards to attenuation of risk. Although ENGAGES has been criticized on several levels, ${ }^{36}$ those concerns have been effectively rebutted. ${ }^{37}$

The results of the ENGAGES study changed our perception of the utility of pEEG-guided care in preventing postoperative delirium. In the context of those results, two different meta-analyses fail to detect an effect. In the first, Vlisides and Avidan ${ }^{38}$ pooled the results from the following studies: 1) Cognitive Dysfunction after Anesthesia (CODA) trial, ${ }^{27}$ 2) Surgery Depth of Anaesthesia Cognitive Outcome (SuDoCo) trial, ${ }^{28}$ 3) Being Awake During Surgery and Anesthesia (BAG-RECALL) study, ${ }^{39}$ and 4) ENGAGES. ${ }^{16}$ In their pooled analysis (which applied a per-protocol approach to the results of the SuDoCo trial), delirium was present in 378/1919 (19.7\%) subjects in the EEG group and 390/1661 (23.5\%) in the non-EEG group; the difference was not statistically significant (hazards ratio (HR), 0.764 (95\% CI, 0.549, 1.061); $\mathrm{P}=0.1061)$. A comparable negative result with the same group of studies was obtained in the meta-analysis by the Perioperative Quality Initiative (POQI) group ${ }^{40}$ who reported a relative risk of $0.80(95 \% \mathrm{CI}, 0.60,1.07$; $\mathrm{P}=$ 0.127). More recently, Sun et al performed a similar analysis, ${ }^{30}$ but expanded their analysis to include the results of Jildenstål et $\mathrm{al}^{26}$ in addition to the four studies included in the meta-analysis by Vlisides and Avidan. In this pooled analysis (which did not apply a per-protocol approach to the SuDoCo trial results), delirium was present in 350/1794 (19.5\%) of subjects in the EEG group and $420 / 1818(23.1 \%)$ of subjects in the non-EEG-guided group, and the difference was not significant (HR, 0.79 (95\% CI, 0.60, 1.05); $\mathrm{P}=0.101$ ). Thus, the conclusion one draws from these analyses is that the data do not provide evidence that pEEG-guided anesthesia care reduces the incidence of delirium in the postoperative period, and is consistent with the recent assessment from the Perioperative Quality Initiative (POQI) 6 workgroup $;^{41}$ it is important to note, however, there was a lack of consensus within the Workgroup as to this conclusion, and those concerns were published online in a separate statement (http://links.lww.com/AA/D5).

More recently, the results of the Anesthetic Depth and Postoperative Delirium Trial-2 (ADAPT-2) trial were published. ${ }^{42}$ Similar to the STRIDE and ENGAGES studies, ADAPT-2 was a single-center RCT, but in contrast, enrolled subjects age $\geq 65$ yr undergoing elective major noncardiac surgery (excluding intracranial procedures). The primary objective of the study was to determine whether the use of a pEEG monitor (SEDline Brain Function Monitor; Masimo) reduced the extent of EEG burst suppression, and the secondary outcome was whether the use of the monitor reduced the incidence of delirium on postoperative days 1 to 3 . Three hundred and eightyone subjects were screened, 223 were randomized - 109 to the EEG-guided care group and 114 to the "standard" care group (EEG monitoring was performed, but the anesthesia team was blind to the data). For subjects in the EEGguided group, the goal was to maintain the patient state index (PSI; a proprietary, dimensionless number (scaled from 0 to 100) that is thought to reflect anesthetic depth, with PSI values of 25-50 commensurate with a surgical plane of anesthesia). Although designed as an intention-totreat study, the final sample sizes in each group were less than the randomized sample sizes due to cancellation of surgery, withdrawal of consent and skin irritation. Thus, in the EEG-guided group, 101 subjects were included in the EEG analysis and 100 subjects were included in the delirium analysis while in the "standardized" care group, 98 subjects were included in the postoperative EEG-analysis with 101 subjects in the delirium analysis. On the whole, the groups were evenly matched except on the measure of preoperative cognitive impairment (as measured by the Telephone Interview for Cognitive Status; TICS), which was more prevalent in the EEG-guided group than in the standardized-care group (13/102 [13\%] and 8/102 [8\%], respectively). Not surprisingly, subjects in the EEG-guided care group spent less time (minutes) in burst suppression than those in the standard-care group (median [IQR]: 4.2 [15.4] and 7.6 [28.3], $p=0.02$ ). In contrast, the incidence of postoperative delirium [as measured by CAM] was unaffected (n [\%]; EEG group - 17 [17\%], Standard group -20 [20\%], $p=0.53$ ). In addition to using CAM to measure the absence/presence of delirium, the 
investigators also used the Memorial Delirium Assessment Scale (MDAS), a validated instrument that measures severity, rather than simply presence, of delirium; ${ }^{43,44}$ as with the CAM scores, the MDAS scores were comparable between the two groups on all days measured. These findings are congruent with the lack of efficacy for pEEGguided anesthesia care as a preventative strategy for reducing postoperative delirium discussed thus far.

This brings us to the recent published study on this topic, that by Pedemonte et al. ${ }^{45}$ Unlike the above studies, this was a retrospective cohort observational sub-study of the Minimizing ICU Neurological Dysfunction with Dexmedetomidine-induced Sleep (MINDDS) trial. As described,

The MINDDS trial is a 370-patient block-randomised, placebo controlled, double-blinded, single-site, parallelarm superiority trial. Patients over 60 years old, undergoing cardiac surgery with planned cardiopulmonary bypass [CPB], will be randomised to receive a sleepinducing dose of dexmedetomidine or placebo. The primary outcome is the incidence of delirium on postoperative day 1, assessed with the Confusion Assessment Method by staff blinded to the treatment assignment. ${ }^{46}$

Of note, intraoperative management was not adjusted based on available intraoperative pEEG (Masimo SEDline) data. In the present study, of 159 patients who underwent surgery, pEEG data were available on 141 . The incidence of delirium in patients with burst-suppression during CPB was $25 \%$ (15 of 60 ) compared with $6.2 \%$ (5 of 81) in patients without burst-suppression during CPB. From a series of univariate logistic regression and causal/ mediational inference analyses, they derived a model in which EEG burst suppression during CPB was a predictor of postoperative delirium, and the likelihood of experiencing burst suppression was a function of physical function, lowest temperature during $\mathrm{CPB}$, and EEG alpha power.

The fact that hypothermia was identified as a dependent variable is notable because hypothermia can induce burst suppression on the EEG, ${ }^{47}$ and although the argument has been made that intentional induction of burst suppression by any means (temperature or anesthetic drug) may offer some degree of neuroprotection against ischemic injury, the evidence in humans supporting this conjecture is poor. ${ }^{48,49}$ Similarly, the correlation with the EEG alpha signal is relevant due to the observation that 1) lower intraoperative frontal alpha power correlates with lower preoperative cognitive function ${ }^{50}$ and 2) a pronounced EEG alpha component during emergence from general anesthesia was associated with less delirium in the recovery room. ${ }^{51}$

With those considerations in mind, the obvious inference of the MINDDS model is that if anesthetic administration is titrated to the alpha signal then it might be possible to reduce the incidence of postoperative delirium in this patient population (of interest, time spent in burst suppression does not correlate with time to emergence or the degree of cognitive impairment upon emergence from anesthesia in healthy adults. ${ }^{52}$ But the data are only correlational, and an appropriately designed study is needed to explicitly address this hypothesis. Importantly, the regression/inference analyses did not account for dexmedetomidine administration, and without unblinding, any meaningful inferences as to causal relationships are questionable.

Another important recent study adds to our understanding of how pEEG monitoring might be useful while also proving insights into the underlying biology of delirium in the postoperative period. ${ }^{53}$ Here, usable EEG data were collected from 70 subjects before and after surgery between 2015 and 2019 who were previously enrolled in either Interventions for Postoperative Delirium: Biomarker-3 (IPOD-B3), a cohort study of adult patients (age $>65 \mathrm{yr}$ ) undergoing major surgery (www.clinical trials.gov number: NCT03124303) or Interventions for Postoperative Delirium: Biomarker-2 (IPOD-B2), a cohort study of adult subjects undergoing thoracic aortic aneurysm repair (www.clinicaltrials.gov number: NCT02926417). Additional measurements included a 10 cytokine panel which consisted of interleukin (IL) 1 (IL-1) $\beta$, IL-1 receptor antagonist, IL-2, IL-4, IL-6, IL-8, IL-10, IL-12, monocyte chemoattractant protein 1 (MCP-1), and tumor necrosis factor (TNF) $\alpha$ and diffusion tensor imaging (DTI). Postoperative delirium was diagnosed using either CAM (if not requiring mechanical ventilation) or CAM-ICU (if mechanical ventilation required) methodology; of the 70 subjects, $22(31.4 \%)$ were diagnosed with delirium and $48(68.6 \%)$ were without delirium. Subjects who experienced postoperative delirium had higher alpha (6-12 Hz bandwidth) power on the preoperative EEG along with increased preoperative alpha band connectivity, but impaired structural connectivity on DTI; postoperatively, these subjects had increased slow wave activity (SWA, which included $\delta, 0.5-4 \mathrm{~Hz}$ and $\theta$ : 4-6 Hz bandwidth activity) in occipitoparietal and frontal cortices 
along with impaired functional connectivity. In contrast, frontal alpha power increased postoperatively in subjects without delirium. Of the cytokines measured, there were statistically significant changes in MCP-1 $\left(\mathrm{r}^{2}=0.192 ; \mathrm{P}=\right.$ $0.002)$ and IL-10 $\left(\mathrm{r}^{2}=0.200 ; \mathrm{P}=0.002\right)$ that correlated with both delirium severity and delirium incidence.

This work validates prior observations that decreased alpha band connectivity may predispose to delirium in the postoperative period ${ }^{54,55}$ and is the basis for the suggestion by the authors that "manipulation of alpha band connectivity may yet prove to be a therapy for delirium.” Implicit in this suggestion is the concept that titrating the dose of anesthetic (volatile or intravenous) to produce the desired
EEG alpha signature will minimize the incidence of POD. Presumably the preoperative EEG characteristics associated with postoperative delirium are unlikely to be easily, or quickly, modified. An important limitation in the present study is the absence of intraoperative EEG data, but this merely provides opportunity for additional studies to determine if it is possible to target intraoperative anesthetic administration to a pre-specified EEG pattern of activity (other than avoiding burst suppression) to achieve the desired outcome. The correlation of EEG alpha, POD and MCP-1 and IL-10 provides a biologic link between inflammatory pathways and the development of POD. Whether the changes in MCP-1 and IL-10 result from anesthesia

Table I Trials Registered with ClinicalTrials.gov Examining the Impact of EEG-Guided Anesthesia Care on Postoperative Delirium

\begin{tabular}{|c|c|c|c|}
\hline $\begin{array}{l}\text { ClinicalTrials. } \\
\text { gov Number }\end{array}$ & Study Title & $\begin{array}{c}\text { Actual/ } \\
\text { Projected } \\
\text { Enrollment }\end{array}$ & Status \\
\hline NCT0I983384 & Anesthetic Depth and Postoperative Delirium Trial - 2 & 205 & $\begin{array}{l}\text { Completed; results } \\
\text { published - Tang et al } \\
2020^{42}\end{array}$ \\
\hline NCT02 133430 & Optimized Anesthesia to Reduce Incidence of Postoperative Delirium & 140 & $\begin{array}{l}\text { Status unknown: Last } \\
\text { update posted: May 12, } \\
2014\end{array}$ \\
\hline NCT02382445 & $\begin{array}{l}\text { Anesthesia Depth Increases the Degree of Postoperative Dementia, } \\
\text { Delirium, and Cognitive Dysfunction (BIS \& Dementia) }\end{array}$ & 138 & $\begin{array}{l}\text { Completed: Last update } \\
\text { posted: May 9, } 2017\end{array}$ \\
\hline NCT02604459 & Does Optimized General Anesthesia Care Reduce Postoperative Delirium? & 160 & $\begin{array}{l}\text { Status unknown: Last } \\
\text { update posted: June I, } \\
2017\end{array}$ \\
\hline NCT02692300 & EEG Guidance of Anesthesia (ENGAGES-CANADA) & 1200 & Recruiting \\
\hline NCT02698982 & $\begin{array}{l}\text { Optimized Anesthesia to Reduce Postoperative Cognitive Impairment in the } \\
\text { Elderly }\end{array}$ & 41 & $\begin{array}{l}\text { Completed: Last update } \\
\text { posted: Dec. } 26,2017\end{array}$ \\
\hline NCT03330236 & EEG - Guided Anesthetic Care and Postoperative Delirium (EMODIPOD) & 1560 & $\begin{array}{l}\text { Completed: Last update } \\
\text { posted: Sept. } 25,2019\end{array}$ \\
\hline NCT03705728 & $\begin{array}{l}\text { Automated Administration of Intravenous Compared With Inhalatory } \\
\text { Anesthesia on the Occurrence of Postoperative Delirium }\end{array}$ & 1000 & Recruiting \\
\hline NCT03706989 & $\begin{array}{l}\text { Predicting Postoperative Delirium Using EEG, Genetics and } \\
\text { Neurobiomarkers of Cerebral Injury (POD-0I) }\end{array}$ & 480 & Recruiting \\
\hline NCT03775356 & Reduction of Intraoperative EEG Burst Suppression (BsR) & 66 & Recruiting \\
\hline NCT04246320 & Taking Brain Monitoring to the Next Level & 60 & Recruiting \\
\hline NCT0429256I & $\begin{array}{l}\text { Intraoperative EEG Monitoring and Postoperative Delirium in Elderly } \\
\text { Patients With Sevoflurane Anesthesia }\end{array}$ & 500 & Recruiting \\
\hline NCT044435I7 & $\begin{array}{c}\text { Modulation Of Frontal EEG Alpha Oscillations During Maintenance and } \\
\text { Emergence Phases of General Anesthesia }\end{array}$ & 600 & Not yet recruiting \\
\hline
\end{tabular}


per se or represent surgical-induced inflammation (or an interaction between the two) is unclear, but previous work has shown that anesthetic administration on its own does not result in an increase in serum inflammatory biomarker (C-reactive protein (CRP), glial fibrillary acidic protein (GFAP), IL-6, neurofilament light (NF-L), and TNF- $\alpha$ ) production. ${ }^{56}$ Adding further nuance to this picture are the results from a single-center placebo-controlled trial of dexamethasone (1 $\mathrm{mg} / \mathrm{kg})$ administered on induction of anesthesia in subjects undergoing cardiopulmonary bypass surgery (the Dexamethasone for Cardiac Surgery (DECS) trial) which failed to demonstrate a reduction in postoperative delirium using the CAM-ICU method. ${ }^{57}$

\section{Conclusion}

Reducing postoperative delirium is a laudable goal. The issue of whether pEEG-guided anesthesia care can reduce postoperative delirium remains hotly contested. ${ }^{58-60}$ It is not a trivial question, and any future recommendations concerning its use should be based on solid evidence. At present, the existing data do not support its routine use for the prevention of postoperative delirium. One could argue for its use on the basis of the "What's the harm?" argument as it is a non-invasive device with no obvious useassociated risk (skin irritation notwithstanding). But this claim fails as there is harm as its use has associated costs (both economic and environmental due to the costs of consumables acquisition and disposal) without evidence of benefit. Consequently, that argument fails a guiding principle of medical ethics, that of nonmaleficence (the requirement to do no harm to the patient), and may fail another ethical principle, that of beneficence (the requirement to provide benefit to the patient), depending on the intended benefit. A Boolean search of interventional clinical trials in adults registered at www.clinicaltrials.gov (search terms: "anesthesia", "postoperative delirium", "EEG") identified a number of trials in various stages of activity (Table 1); six are large, with target enrollments $\geq$ 500 , and collectively these studies will seek to enroll 6150 subjects. While no one study will likely answer the question, the studies in aggregate may provide clearer insight into which patients, if any, will benefit from this intervention. In the meantime, pEEG-guided anesthesia care may be reasonable if the goal is to facilitate rapid emergence and recovery ${ }^{61-64}$ and certainly in the context of welldesigned clinical trials.

The answer to the question of whether pEEG-directed minimization of anesthetic exposure will likely lead to a reduction in POD is unknown. The answer will likely hinge on a more sophisticated understanding of the interrelationship of EEG signatures during the conscious state and during anesthetic-induced loss of consciousness. ${ }^{65-67}$ Similarly, merely relying on the pattern of activity obtained from sampling the frontal EEG may well be insufficient ${ }^{68}$ given the role connectivity plays in predicting POD as demonstrated by Tanabe and colleagues, ${ }^{53}$ and the next generation of pEEG monitors should incorporate the ability to measure posterior electrical activity. Inflammation, and by extension, neuroinflammation, is almost certainly linked to the more protracted phenomenon of postoperative cognitive dysfunction, ${ }^{69}$ but what role it plays in the short-term ontogeny of delirium is less clear, and disentangling the influence of surgical-induced inflammation on the development of postoperative delirium and postoperative NCD will be an ongoing challenge.

\section{Acknowledgments}

This work was supported by the Department of Anesthesiology, Weill Cornell Medicine. One author (PAG) receives additional support from the National Institutes of Health and Akelos, Inc. (New York, NY) for research efforts unrelated to this study.

\section{Disclosure}

The authors report no conflicts of interest in this work.

\section{References}

1. Moller JT, Cluitmans P, Rasmussen LS, et al. Long-term postoperative cognitive dysfunction in the elderly ISPOCD1 study. ISPOCD investigators. international study of post-operative cognitive dysfunction. Lancet. 1998;351(9106):857-861. doi:10.1016/S0140-6736(97)07382-0

2. Evered L, Silbert B, Knopman DS, et al. Recommendations for the nomenclature of cognitive change associated with anaesthesia and surgery-2018. Br J Anaesth. 2018;121(5):1005-1012. doi:10.1016/j.bja.2017.11.087

3. Scott DA, Evered L, Maruff P, MacIsaac A, Maher S, Silbert BS. Cognitive function before and after left heart catheterization. $J \mathrm{Am}$ Heart Assoc. 2018;7(6):6. doi:10.1161/JAHA.117.008004

4. Evered LA, Silbert BS, Scott DA, Maruff P, Ames D. Prevalence of dementia 7.5 years after coronary artery bypass graft surgery. Anesthesiology. 2016;125(1):62-71. doi:10.1097/ALN.000000000 0001143

5. Inouye SK. Delirium in hospitalized older patients: recognition and risk factors. J Geriatr Psychiatry Neurol. 1998;11(3):118-125. doi:10.1177/089198879801100302

6. Ely EW, Inouye SK, Bernard GR, et al. Delirium in mechanically ventilated patients: validity and reliability of the confusion assessment method for the intensive care unit (CAM-ICU). JAMA. 2001;286 (21):2703-2710. doi:10.1001/jama.286.21.2703

7. Marcantonio ER, Ngo LH, O'Connor M, et al. 3D-CAM: derivation and validation of a 3-minute diagnostic interview for CAM-defined delirium: a cross-sectional diagnostic test study. Ann Intern Med. 2014;161(8):554-561. doi:10.7326/M14-0865 
8. Wong CL, Holroyd-Leduc J, Simel DL, Straus SE. Does this patient have delirium? Value of bedside instruments. JAMA. 2010;304 (7):779-786. doi:10.1001/jama.2010.1182

9. Rudolph JL, Marcantonio ER. Review articles: postoperative delirium: acute change with long-term implications. Anesth Analg 2011;112(5):1202-1211. doi:10.1213/ANE.0b013e3182147f6d

10. Pezzullo L, Streatfeild J, Hickson J, Teodorczuk A, Agar MR, Caplan GA. Economic impact of delirium in Australia: a cost of illness study. BMJ Open. 2019;9(9):e27514. doi:10.1136/bmjopen2018-027514

11. Hshieh TT, Inouye SK, Oh ES. Delirium in the elderly. Psychiatr Clin North Am. 2018;41(1):1-17. doi:10.1016/j.psc.2017.10.001

12. Weir E, O'Brien AJ. Don't go there - it's not a nice place: older adults' experiences of delirium. Int J Ment Health Nurs. 2019;28 (2):582-591. doi:10.1111/inm.12563

13. Evered LA, Silbert BS. Postoperative cognitive dysfunction and noncardiac surgery. Anesth Analg. 2018;127(2):496-505. doi:10.1213/ANE.0000000000003514

14. Neufeld KJ, Thomas C. Delirium: definition, epidemiology, and diagnosis. J Clin Neurophysiol. 2013;30(5):438-442. doi:10.1097/ WNP.0b013e3182a73e31

15. Parrish E. Delirium superimposed on dementia: challenges and opportunities. Nurs Clin North Am. 2019;54(4):541-550. doi:10.1016/j.cnur.2019.07.004

16. Wildes TS, Mickle AM, Ben Abdallah A, et al. Effect of electroencephalography-guided anesthetic administration on postoperative delirium among older adults undergoing major surgery: the ENGAGES randomized clinical trial. JAMA. 2019;321 (5):473-483. doi:10.1001/jama.2018.22005

17. Gibbs FA, Gibbs LE, Lennox WG. Effects on the electroencephalogram of certain drug which influence nervous activity. Arch Intern Med. 1937;60(1):154-166. doi:10.1001/archinte.1937.0018001 0159012

18. Findeiss JC, Kien GA, Huse KO, Linde HW. Power spectral density of the electroencephalogram during halothane and cyclopropane anesthesia in man. Anesth Analg. 1969;48(6):1018-1023. doi:10.1213/00000539-196911000-00027

19. Bart AJ, Homi J, Linde HW. Changes in power spectra of electroencephalograms during anesthesia with fluroxene, methoxyflurane and ethrane. Anesth Analg. 1971;50(1):53-63. doi:10.1213/ 00000539-197101000-00011

20. Bickford RG, Fleming N, Billinger T. Compression of EEG data. Trans Am Neurol Assoc. 1971;96:118-122.

21. Myers RR, Stockard JJ, Fleming NI, France CJ, Bickford RG. The use of on-line telephonic computer analysis of the E.E.G. in anaesthesia. Br J Anaesth. 1973;45(7):664-670. doi:10.1093/bja/ 45.7.664

22. Fleming RA, Smith NT. An inexpensive device for analyzing and monitoring the electroencephalogram. Anesthesiology. 1979;50 (5):456-460. doi:10.1097/00000542-197905000-00014

23. Fritz BA, Kalarickal PL, Maybrier HR, et al. Intraoperative electroencephalogram suppression predicts postoperative delirium. Anesth Analg. 2016;122(1):234-242. doi:10.1213/ANE.0000000000000989

24. Berger M, Schenning KJ, Brown C, et al. Best practices for postoperative brain health: recommendations from the fifth international perioperative neurotoxicity working group. Anesth Analg. 2018;127 (6):1406-1413. doi:10.1213/ANE.0000000000003841

25. Mahanna-Gabrielli E, Schenning KJ, Eriksson LI, et al. State of the clinical science of perioperative brain health: report from the American society of anesthesiologists brain health initiative summit 2018. $B r \quad J$ Anaesth. 2019;123(4):464-478. doi:10.1016/j. bja.2019.07.004

26. Jildenstål PK, Hallén JL, Rawal N, Berggren L. Does depth of anesthesia influence postoperative cognitive dysfunction or inflammatory response following major ENT surgery? J Anesth Clin Res. 2012;3(6):220. doi:10.4172/2155-6148.1000220
27. Chan MT, Cheng BC, Lee TM, Gin T, Group CT. BIS-guided anesthesia decreases postoperative delirium and cognitive decline. $J$ Neurosurg Anesthesiol. 2013;25(1):33-42. doi:10.1097/ ANA.0b013e3182712fba

28. Radtke FM, Franck M, Lendner J, Kruger S, Wernecke KD, Spies CD. Monitoring depth of anaesthesia in a randomized trial decreases the rate of postoperative delirium but not postoperative cognitive dysfunction. Br J Anaesth. 2013;110(Suppl 1):i98-105. doi:10.1093/bja/aet055

29. Whitlock EL, Villafranca AJ, Lin N, et al. Relationship between bispectral index values and volatile anesthetic concentrations during the maintenance phase of anesthesia in the B-Unaware trial. Anesthesiology. 2011;115(6):1209-1218. doi:10.1097/ALN.0b013e3182395dcb

30. Sun Y, Ye F, Wang J, et al. Electroencephalography-guided anesthetic delivery for preventing postoperative delirium in adults: an updated meta-analysis. Anesth Analg. 2020;131(3):712-719. doi:10.1213/ ANE.0000000000004746

31. Brown C, Laflam A, Max L, et al. The impact of delirium after cardiac surgical procedures on postoperative resource use. Ann Thorac Surg. 2016;101(5):1663-1669. doi:10.1016/j.athoracsur.20 15.12.074

32. Neufeld KJ, Leoutsakos JM, Sieber FE, et al. Outcomes of early delirium diagnosis after general anesthesia in the elderly. Anesth Analg. 2013;117(2):471-478. doi:10.1213/ANE.0b013e3182973650

33. Lingehall HC, Smulter NS, Lindahl E, et al. Preoperative cognitive performance and postoperative delirium are independently associated with future dementia in older people who have undergone cardiac surgery: a longitudinal cohort study. Crit Care Med. 2017;45 (8):1295-1303. doi:10.1097/CCM.0000000000002483

34. Sieber FE, Neufeld KJ, Gottschalk A, et al. Effect of depth of sedation in older patients undergoing hip fracture repair on postoperative delirium: the STRIDE randomized clinical trial. JAMA Surg. 2018;153(11):987-995. doi:10.1001/jamasurg.2018.2602

35. van Velthuijsen EL, Zwakhalen SM, Warnier RM, Mulder WJ, Verhey FR, Kempen GI. Psychometric properties and feasibility of instruments for the detection of delirium in older hospitalized patients: a systematic review. Int $J$ Geriatr Psychiatry. 2016;31 (9):974-989.

36. Koch S, Radtke F, Spies C. A call for a more rigorous screening of postoperative delirium. Ann Transl Med. 2019;7(Suppl 6):S192. doi:10.21037/atm.2019.07.32

37. Wildes TS, Avidan MS. Critical appraisal of ENGAGES: cognitive dissonance and anesthesia research. Ann Transl Med. 2019;7(20):599. doi:10.21037/atm.2019.09.48

38. Vlisides P, Avidan M. Recent advances in preventing and managing postoperative delirium. F1000Res. 2019;8.

39. Whitlock EL, Torres BA, Lin N, et al. Postoperative delirium in a substudy of cardiothoracic surgical patients in the BAG-RECALL clinical trial. Anesth Analg. 2014;118(4):809-817. doi:10.1213/ ANE.0000000000000028

40. Chan MTV, Hedrick TL, Egan TD, et al. American society for enhanced recovery and perioperative quality initiative joint consensus statement on the role of neuromonitoring in perioperative outcomes: electroencephalography. Anesth Analg. 2020;130(5):1278-1291. doi:10.1213/ANE.0000000000004502

41. Hughes CG, Boncyk CS, Culley DJ, et al. American society for enhanced recovery and perioperative quality initiative joint consensus statement on postoperative delirium prevention. Anesth Analg. 2020;130(6):1572-1590. doi:10.1213/ANE.0000000000004641

42. Tang CJ, Jin Z, Sands LP, et al. ADAPT-2: a randomized clinical trial to reduce intraoperative EEG suppression in older surgical patients undergoing major noncardiac surgery. Anesth Analg. 2020;131 (4):1228-1236. doi:10.1213/ANE.0000000000004713

43. Breitbart W, Rosenfeld B, Roth A, Smith MJ, Cohen K, Passik S. The memorial delirium assessment scale. J Pain Symptom Manage. 1997;13(3):128-137. doi:10.1016/S0885-3924(96)00316-8 
44. Jones RN, Cizginer S, Pavlech L, et al. Assessment of instruments for measurement of delirium severity: a systematic review. JAMA Intern Med. 2019;179(2):231-239. doi:10.1001/jamainternmed.2018.6975

45. Pedemonte JC, Plummer GS, Chamadia S, et al. Electroencephalogram burst-suppression during cardiopulmonary bypass in elderly patients mediates postoperative delirium. Anesthesiology. 2020;133 (2):280-292. doi:10.1097/ALN.0000000000003328

46. Shelton KT, Qu J, Bilotta F, et al. Minimizing ICU neurological dysfunction with dexmedetomidine-induced sleep (MINDDS): protocol for a randomised, double-blind, parallel-arm, placebo-controlled trial. BMJ Open. 2018;8(4):e020316. doi:10.1136/bmjopen-2017-020316

47. Westover MB, Ching S, Kumaraswamy VM, et al. The human burst suppression electroencephalogram of deep hypothermia. Clin Neurophysiol. 2015;126(10):1901-1914. doi:10.1016/j.clinph. 2014.12.022

48. Roach GW, Newman MF, Murkin JM, et al. Ineffectiveness of burst suppression therapy in mitigating perioperative cerebrovascular dysfunction. Multicenter study of perioperative ischemia (McSPI) research group. Anesthesiology. 1999;90(5):1255-1264. doi:10.1097/00000542-199905000-00006

49. Hindman BJ, Bayman EO, Pfisterer WK, Torner JC, Todd MM, Investigators I. No association between intraoperative hypothermia or supplemental protective drug and neurologic outcomes in patients undergoing temporary clipping during cerebral aneurysm surgery: findings from the intraoperative hypothermia for aneurysm surgery trial. Anesthesiology. 2010;112(1):86-101. doi:10.1097/ALN.0b0 $13 \mathrm{e} 3181 \mathrm{c} 5 \mathrm{e} 28 \mathrm{f}$

50. Giattino CM, Gardner JE, Sbahi FM, et al. Intraoperative frontal alpha-band power correlates with preoperative neurocognitive function in older adults. Front Syst Neurosci. 2017;11:24. doi:10.3389/ fnsys.2017.00024

51. Hesse S, Kreuzer M, Hight D, et al. Association of electroencephalogram trajectories during emergence from anaesthesia with delirium in the postanaesthesia care unit: an early sign of postoperative complications. Br J Anaesth. 2019;122(5):622-634. doi:10.1016/j. bja.2018.09.016

52. Shortal BP, Hickman LB, Mak-McCully RA, et al. Duration of EEG suppression does not predict recovery time or degree of cognitive impairment after general anaesthesia in human volunteers. $\mathrm{Br}$ J Anaesth. 2019;123(2):206-218. doi:10.1016/j.bja.2019.03.046

53. Tanabe S, Mohanty R, Lindroth $\mathrm{H}$, et al. Cohort study into the neural correlates of postoperative delirium: the role of connectivity and slow-wave activity. Br J Anaesth. 2020;125(1):55-66. doi:10.1016/j. bja.2020.02.027

54. van Dellen E, van der Kooi AW, Numan T, et al. Decreased functional connectivity and disturbed directionality of information flow in the electroencephalography of intensive care unit patients with delirium after cardiac surgery. Anesthesiology. 2014;121(2):328-335. doi:10.1097/ALN.0000000000000329

55. Numan T, Slooter AJC, van der Kooi AW, et al. Functional connectivity and network analysis during hypoactive delirium and recovery from anesthesia. Clin Neurophysiol. 2017;128(6):914-924. doi:10.1016/j.clinph.2017.02.022
56. Deiner S, Baxter MG, Mincer JS, et al. Human plasma biomarker responses to inhalational general anaesthesia without surgery. $\mathrm{Br}$ J Anaesth. 2020;125(3):282-290. doi:10.1016/j.bja.2020.04.085

57. Sauër AM, Slooter AJ, Veldhuijzen DS, van Eijk MM, Devlin JW, van Dijk D. Intraoperative dexamethasone and delirium after cardiac surgery: a randomized clinical trial. Anesth Analg. 2014;119 (5):1046-1052. doi:10.1213/ANE.0000000000000248

58. Whitlock EL, Avidan MS. Three blind mice: a tail of discordant trials. $B r \quad J$ Anaesth. 2020;124(2):121-125. doi:10.1016/j. bja.2019.09.035

59. Berger M, Mark JB, Kreuzer M. Of parachutes, speedometers, and EEG: what evidence do we need to use devices and monitors? Anesth Analg. 2020;130(5):1274-1277. doi:10.1213/ANE.0000000000004653

60. Garcia PS. Meta-analysis and megadata in electroencephalogram-based techniques for delirium prevention. Anesth Analg. 2020;131(3):709-711. doi:10.1213/ANE.000000000 0004867

61. Johansen JW, Sebel PS, Sigl JC. Clinical impact of hypnotic-titration guidelines based on EEG bispectral index (BIS) monitoring during routine anesthetic care. J Clin Anesth. 2000;12(6):433-443. doi:10.1016/S0952-8180(00)00187-2

62. Wong J, Song D, Blanshard H, Grady D, Chung F. Titration of isoflurane using BIS index improves early recovery of elderly patients undergoing orthopedic surgeries. Can J Anaesth. 2002;49 (1):13-18. doi:10.1007/BF03020413

63. White PF, Ma H, Tang J, Wender RH, Sloninsky A, Kariger R. Does the use of electroencephalographic bispectral index or auditory evoked potential index monitoring facilitate recovery after desflurane anesthesia in the ambulatory setting? Anesthesiology. 2004;100 (4):811-817. doi:10.1097/00000542-200404000-00010

64. Liao WW, Wang JJ, Wu GJ, Kuo CD. The effect of cerebral monitoring on recovery after sevoflurane anesthesia in ambulatory setting in children: a comparison among bispectral index, A-line autoregressive index, and standard practice. J Chin Med Assoc. 2011;74 (1):28-36. doi:10.1016/j.jcma.2011.01.004

65. Lee M, Sanders RD, Yeom SK, et al. Network properties in transitions of consciousness during propofol-induced sedation. Sci Rep. 2017;7(1):16791. doi:10.1038/s41598-017-15082-5

66. Darracq M, Funk CM, Polyakov D, et al. Evoked alpha power is reduced in disconnected consciousness during sleep and anesthesia. Sci Rep. 2018;8(1):16664. doi:10.1038/s41598-018-34957-9

67. Sanders RD, Banks MI, Darracq M, et al. Propofol-induced unresponsiveness is associated with impaired feedforward connectivity in cortical hierarchy. $B r \quad J$ Anaesth. 2018;121(5):1084-1096. doi:10.1016/j.bja.2018.07.006

68. Sanders RD, Mostert N, Lindroth H, Tononi G, Sleigh J. Is consciousness frontal? Two perioperative case reports that challenge that concept. $B r \quad J$ Anaesth. 2018;121(1):330-332. doi:10.1016/j. bja.2018.01.010

69. Safavynia SA, Goldstein PA. The role of neuroinflammation in postoperative cognitive dysfunction: moving from hypothesis to treatment. Front Psychiatry. 2018;9:752. doi:10.3389/fpsyt.2018.00752

\section{Publish your work in this journal}

The International Journal of General Medicine is an international, peer-reviewed open-access journal that focuses on general and internal medicine, pathogenesis, epidemiology, diagnosis, monitoring and treatment protocols. The journal is characterized by the rapid reporting of reviews, original research and clinical studies across all disease areas. The manuscript management system is completely online and includes a very quick and fair peer-review system, which is all easy to use. Visit http://www.dovepress.com/ testimonials.php to read real quotes from published authors. 